\title{
Design of Dual-mode RFID Reader
}

\author{
Yulin Zhang, Tongtong Wang, Changbin Shang \\ Department of Computer Science and Technology, University of Jinan, Jinan, 250022, China \\ tongtong_6861@126.com
}

\begin{abstract}
The Internet of Things accelerates the development of RFID technology. Logistics, transportation, aviation and other fields has increasingly high demand on the reader's identification distance and reliability. In order to meet the market demand, the design of RFID reader based on the dualmode was proposed. One is $915 \mathrm{MHz}$ radio frequency identification mode. The other is $2.4 \mathrm{GHz}$ active radio frequency identification mode, which has lower transmission power requirement and farther valid identification distance. An ARM9 microcontroller is used as the main controller of the RFID reader. The design includes communicate with PC, control dual-mode RFID reader, control the reader to write and read tags and the study of anti-collision algorithm. In the testing of the system, it achieved to identify further. The performance is achieved and reliable.
\end{abstract}

Keywords-RFID; reader; dual-mode; the Internet of things

\section{INTRODUCTION}

Internet of Things has recently become a research focus. As the key technology, RFID (Radio Frequency Identification) is a non-contact automatic identification technology that uses radio-frequency electromagnetic fields to transfer data. Because of its characteristics include long read-distance, quick speed, strong permeability and bearing the environment, it is widely used in many fields, such as transportation, logistics, aviation, identification and manufacturing $^{[1]}$, etc. Divided in accordance with their radio frequency, there are low-frequency (125 or 134.2 $\mathrm{kHz}$ ), high frequency (13.56 MHz), UHF (868 $956 \mathrm{MHz}$ ) and microwave $(2.4 \mathrm{GHz})$ in the RFID products ${ }^{[2]}$. UHF $(915 \mathrm{MHz})$ is achieved mainly through the capacitive coupling. It can manner to achieve a good reading distance, but is difficult to define the reading area. Active RFID technology $(2.4 \mathrm{GHz})$ has the characteristics of low transmit power, long distance communication, good compatibility and high reliability. Compared with passive RFID, the advantage is very obvious. Complicated as transportation, logistics network, the demand for dual-mode radio frequency technology is urgent. It has broad market prospects.

\section{SYSTEM GENERAL STRUCTURE}

In the design of the dual-mode RFID reader, embedded system design concepts and methods were used. The system is made up of hardware and software. The hardware is the platform that achieves system operation, including a processor and various peripheral interfaces; software controls the system operation, including hardware driver, operating system and various applications. ARM9-based controller can complete the reading and writing of the $915 \mathrm{MHz}$ and $2.4 \mathrm{GHz}$ radio frequency (RF) signal, and the system general diagram is shown in Figure 1.

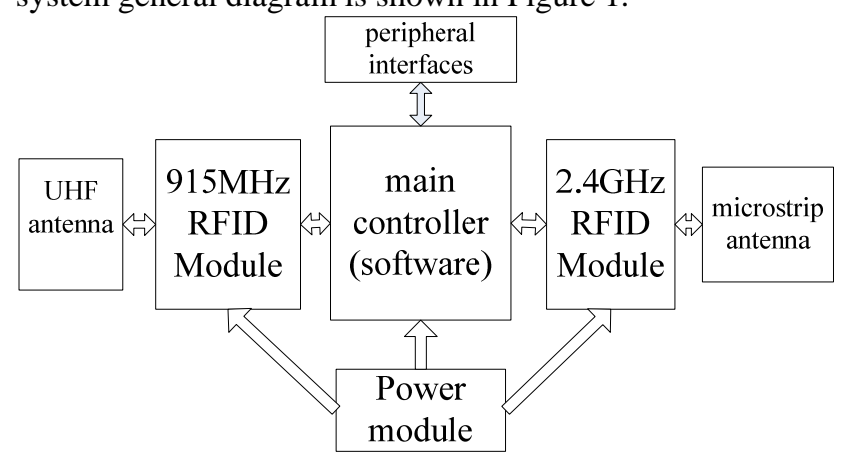

Figure 1. The system general diagram

UHF antenna and the microstrip antenna respectively receive the $915 \mathrm{MHz}$ and $2.4 \mathrm{GHz}$ electromagnetic waves and communicate with the tags. The normal work of the main controller and RF module was provided energy by power module. The $915 \mathrm{MHz}$ RFID modules and $2.4 \mathrm{GHz}$ RFID modules complete RF signal modulation and demodulation, coding and decoding, signal amplification and the transmission and reception, etc. Main controller equipped with the operating system controls the RF signal to read and write. The reader transmits energy through the antenna and receives the return data; Then the received data is sent to the controller for processing; Finally, complete control and read-write operation of the dual mode RF signal.

\section{SYSTEM HARDWARE DESIGN}

\section{A. $915 \mathrm{MHz}$ RFID module}

The design selected RF transceiver chip AS3992 developed by the ams. Integrated with UHF RFID RF frontend and the ISO/I EC18000-6C protocol processor, including a series of advanced features, such as programmable DRM filters, pre-distortion function and the $-86 \mathrm{dBm}$ ultra-high receive sensitivity ${ }^{[3]}$, the device is suitable for a wide range of UHF RFID applications. Parallel or serial interface can be selected for communication between the MCU and the reader.

The $915 \mathrm{MHz}$ reader module can be divided into a transmitter circuit, signal isolation, and the reception circuit, wherein the transmitting circuit was mainly composed of 
AS3992 output matching circuit, the power amplification circuit and a filter circuit. The $915 \mathrm{MHz}$ RF front-end module is shown in Figure 2.

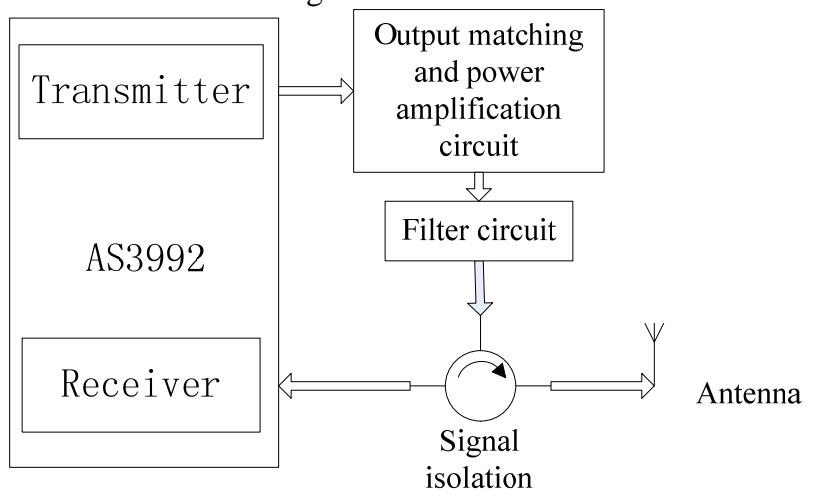

Figure 2. 915MHz RF front-end module

AS3992 internal transmitter can output power to $20 \mathrm{dBm}$.Owing to the recognition distance is short, the chip RF5110 is selected as a power amplifier. The RF5110 is a high-power, high-efficiency power amplifier module, and it can operate in the frequency range $800 \mathrm{MHz}-950 \mathrm{MHz}$. Its Output Power is $+36 \mathrm{dBm}$ at $3.5 \mathrm{~V}$.

915MHz RFID reader needs two antennas to send and receive or transceiver isolation circuit. A directional coupler circuit was used as an isolation circuit for cost savings. Select 5dB directional coupler RCP890A05 as transceiver isolation circuit. Its operating frequency is about 815$960 \mathrm{MHz}$; insertion loss is about $0.19 \mathrm{~dB}$; signal isolation is about 26dB ${ }^{[4]}$. The output signal of amplifier was transmitted to the signal isolation and the reception circuit through the low pass filter. As is shown in Figure 3, transmit power is $30.11 \mathrm{dBm}$ measured with the spectrum analyzer.

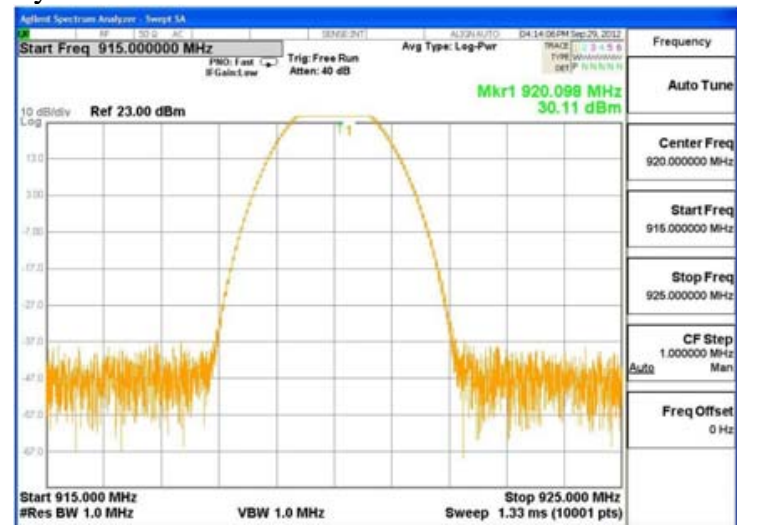

Figure 3. The transmit power spectrogram

\section{B. $2.4 \mathrm{GHz}$ RFID module}

The design select RF transceiver chip NRF24L01 developed by Nordic as $2.4 \mathrm{GHz}$ transceiver. NRF24L01 is a new single chip radio transceiver for the world wide 2.4 -
2.5 GHz ISM band. The transceiver consists of a fully integrated frequency synthesizer, a crystal oscillator, a power amplifier, a demodulator, modulator and Enhanced ShockBurst ${ }^{\mathrm{TM}}$ protocol engine ${ }^{[5]}$. What's more, output power and communication channels can be configured through the program. The power consumption is very low, only $9.0 \mathrm{~mA}$ at an output power of $-6 \mathrm{dBm}$ and $12.3 \mathrm{~mA}$ in RX mode. Built-in Power Down and Standby modes makes power saving easily realizable.

2.4GHz RF module can be divided RF matching and power amplification circuit, a low pass filter, signal isolation, and the reception circuit section. Select ASX621 as a power amplifier to increase the identification distance. ASX621 has the characteristics of high linearity, high gain, and high efficiency in a wide frequency range. The amplified signal was transmitted to the filter and signal isolator to achieve the read-write of the RF signal. The operating circuit is shown in Figure 4.

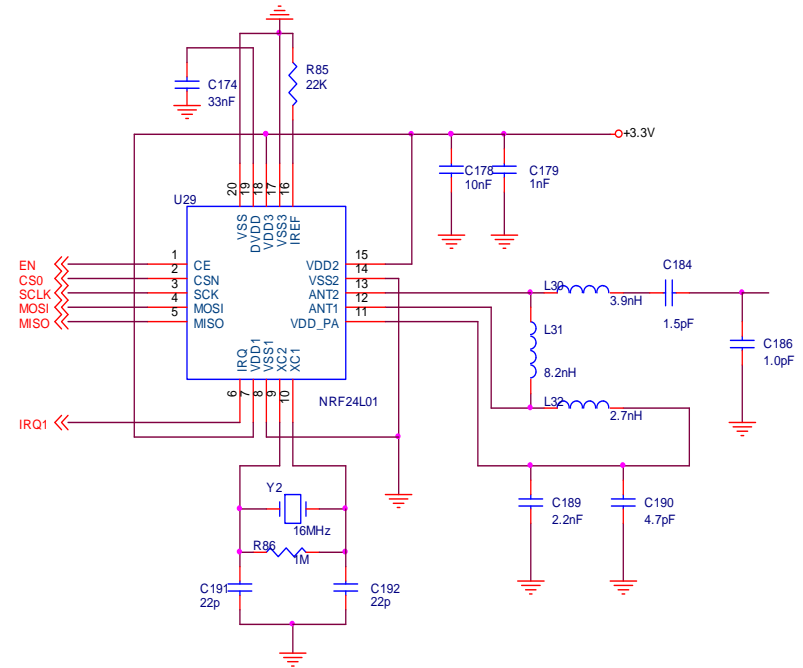

Figure 4. The NRF 24L01 operating circuit

\section{Antenna Selection}

The antenna can transmit radio frequency signal between tags and readers. It works using what's known as "backscatter technology", and its mode is similar to radar. $\mathrm{RF}$ signal generated by the reader radiates to space in a certain direction from the antenna. Through consulting literature, the reader antenna receives power formula is as follows:

$$
P_{\text {收 }}=\frac{\mathrm{P} * G^{2} * \lambda^{2} * \sigma}{(4 p i)^{2} * R^{4}}
$$

In the formula, $\mathrm{P}$ means the transmitting power of the reader, $\mathrm{G}$ is the antenna gain, $\mathrm{R}$ is spacing between tags and the antenna, $\sigma$ means ability test degrees of target transmitting electromagnetic wave. If you want to increase the read-write distance, you can increase the transmission power, the size of antenna reception and the gain. 
When Reader frequency is $915 \mathrm{MHz}$, the polarized microstrip antenna can be used in order to increase the recognition distance. The air is as media; pcb board is as the microstrip ground plane; the radiating portion is the square copper plates, and it respectively feed electricity from the midpoint of each side. When working in the $2.4 \mathrm{GHz}$ you can choose microstrip antenna if you want to have a certain communication distance. Microstrip antenna is a kind of antenna that on a thin dielectric substrate, one side was enclosed the metal thin layer as a ground plate; the other surface was made into a certain shape of the metal patch by the method of photolithography etching, and feeding electricity by using a microstrip line ${ }^{[6]}$.

\section{IV.SYSTEM SOFTWARE DESIGN}

The reader in reading and writing tags needs to switch in the two operating modes. The demands on processor efficiency and processing speed will get more pressing. Two RF chips communicate with the master controller through SPI serial interface. After receive the communication command from the controller, the RF chips notify the controller that there's data to be processed by using IRQ. The transmission and reception of RF signals can be achieved through the operation of the RF chip registers. For system task scheduling and management of system resources, it is necessary to build uCOS- II operating system on the hardware platform. On this basis, completing the tag reading and writing will enable the development of software more convenient and efficient, and the full play of the processing capability of the controller will. The software design includes hardware initialization, the $915 \mathrm{MHz}$ RF's read-write, $2.4 \mathrm{GHz}$ RF's read-write and multi-tag anticollision processing.

For the multi-task scheduling, to achieve inter-task communication and synchronization, semaphore and message queues of uCOS-II system can be used. First, establish the $915 \mathrm{MHz}$ and $2.4 \mathrm{GHz}$ RF module "notice" and "answer" semaphore to achieve synchronization between tasks, and then re-establish message queues which include a certain number of messages for inter-task communication. The software general flowchart is as shown in Figure 5.

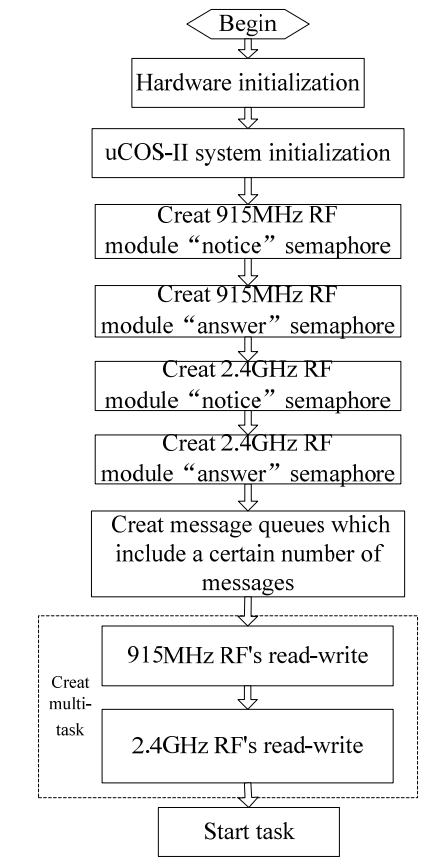

Figure 5. The software general flowchart

\section{A. $915 \mathrm{MHz} R$ R's read-write}

AS3992's initialization is mainly to complete the configuration of work frequency, modulation parameters and chip register through the parallel interface. AS3992 supports multiple protocols, and the ISO6C protocol [7] working mode is selected. The decoding and check had been completed, MCU only need to receive data by parallel or serial interfaces. The Reader identifies all tags though several steps as follows:

1) Set the Q parameter values;

2) The "select" command is issued to select the label group;

3) Start the inventory cycle;

4) Constantly send "QueryRep" and "ACK" commands till all slots of $Q$ value parameter were traversed;

5) Identify all the tags.

\section{B. 2.4GHz RF's read-write}

NRF2401 is a highly integrated RF transceiver chip. Its communication protocols have solidified into chips to simplify the development process. Four operating modes including transceiver mode, configuration mode, idle mode and off mode operating mode are determined by PWR_UP register, PRIM_RX register and CE. The ShockBurstTM transceiver mode was selected. In this operating mode, the programming of the system is simple and higher stability. First initialize transceiver port, and then examine whether the received data is correct or not. After that the tags were coded and decoded, and you need anti-collision processing if there are more tags. All configuration of NRF2401 is done via the SPI. NRF2401 initialization is mainly to configure 
RF transceiver module mode, the frequency of the channel, the transmission data rate, CRC checksum, crystal frequency, address, etc. The initialization code is as follows: void init_NRF24L01(void)

\{

$$
\text { inerDelay(100); }
$$

$\mathrm{CE}=0$;

$\mathrm{CSN}=1$;

$\mathrm{SCK}=0$;

SPI_Write_Buf(WRITE_REG + TX_ADDR,

TX_ADDRESS, TX_ADR_WIDTH);

SPI_Write_Buf(WRITE_REG + RX_ADDR_P0, RX_ADDRESS, RX_ADR_WIDTH);

SPI_RW_Reg(WRITE_REG + EN_AA, x01);

SPI_RW_Reg(WRITE_REG + N_RXADDR, 0x01);

SPI_RW_Reg(WRITE_REG + RF_CH, 0);

SPI_RW_Reg(WRITE_REG +RX_PW_P0, PLOAD_ WIDTH);

$$
\text { SPI_RW_Reg(WRITE_REG + RF_SETUP, 0x07); }
$$

\}

That is, NRF2401 works on the ShockBurstTM transceiver mode, channel frequency is $2.4 \mathrm{GHz}$, transmitting power is $1 \mathrm{MHZ}$, transmitting power is the maximum of $0 \mathrm{~dB}$, and the length of received data is set to 32 bytes.

\section{EXPERIMENTAL RESULTS}

First hardware circuit was tested with a multimeter, oscilloscope, etc. After testing, the welding of the chip device pins is no short-circuit and virtual welding, chip operating voltage and the supply voltage is normal, and transmit power is about $30.11 \mathrm{dBm}$ measured with the spectrum analyzer. In software, the identification distance of the reader and multiple tags read-write were tested. The identification distance of the reader was more than five meters in the system. Multi-tag anti-collision is effect, and the system is stable and reliable. The result of multi-tag reading is as shown in Figure 6, it shows the tags reading times and tag number.

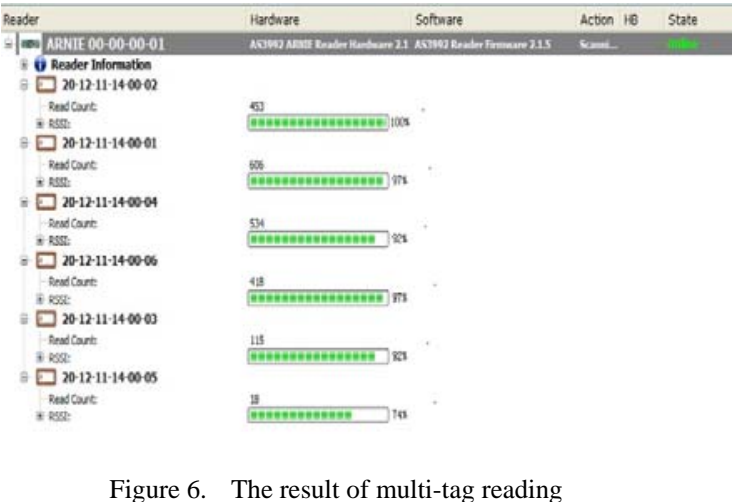

\section{CONCLUSION}

The RFID reader based on dual-mode is put forward, and the hardware organization and software design of the system are also offered in detail. Being tested, it completes basic read and write operations of the dual-mode radio frequency signal. The identification distance is more than $5 \mathrm{~m}$. Besides, the reader has a certain anti-collision ability. It basically achieves the design goals, and has broad market prospects.

\section{ACKNOWLEDGMENT}

I would like to extend my sincere gratitude to Tongtong Wang and Changbin Shang, for their hard work on my thesis. I am deeply grateful of their help in the completion of this thesis.

\section{REFERENCES}

[1] Xiaolin Jia, et al. "RFID Technology and Its Applications in Internet of Things (IOT)," Consumer Electronics, Communications and Networks (CECNet 12), IEEE Press, April 2012, pp. 21-23.

[2] Jeiyoung Lee, et al. "A UHF Mobile RFID Reader IC with SelfLeakage Canceller,” Analog Devices, Inc., Gyeonggi-do, Korea, vol. 02, pp. 273-276, June 2007.

[3] “AS3992 UHF RFID Reader IC”. http://www.ams.com/eng/content/ advancedsearch/AS3992_Datasheet_1V1.pdf.

[4] “RCP890A05_Rev1”. http://wenku.baidu.com/view/96ea1374f46527 d3240ce00d.html.

[5] “NRF24L01”.http://www.nordicsemi.com/eng/Products/2.4GHz-RF/ nRF24L01.

[6] Indrasen Singh, Dr. V.S. Tripathi, "Micro strip Patch Antenna and its Applications: a Survey,” Int. J. Comp. Tech. Appl., Vol 2, pp.15951599, 2011.

[7] ISO/IEC 18000-6C, "Radio-frequency identification for item management-Part 6: Pa rameters for air interface communications at $860 \mathrm{MHz}$ to $960 \mathrm{MHz}$,” 2007. 